Research Article

\title{
Influence of the magnetic fields on some characteristics of raw milk
}

\author{
Hanen Ben Hassen ${ }^{1}$, Anis Elaoud ${ }^{2,3}{ }^{*}$ and Ismail Trabelsi ${ }^{2}$ \\ ${ }^{1}$ Laboratory of probabilities and statistic, Faculty of Sciences of Sfax, Sfax University, Tunisia \\ ${ }^{2}$ Laboratory of Treatment and Recycle of Wastewater, Water Research and Technologies Center -BorjCedria, Tunisia \\ ${ }^{3}$ Laboratory of Environmental Science and Technologies, Higher Institute of Sciences and Technology of Environment Borj Cedraia, Carthage \\ University, Tunisia
}

Received 30 Aug, Accepted 01 Dec 2017, Available online 12 Dec 2017, Vol.5, No.4 (Dec 2017)

\begin{abstract}
Application of magnetic treatment on water and plants has already been shown considerable. A static magnetic treatment of 3300 Gauss strength was conducted for 1 hour on fresh non-sterilized milk and some chemical parameters and microbial effect were followed through the time. The results showed that the magnetic treatment preserved the $\mathrm{pH}$ and the acidity of the milk, however it reduced its conductivity and in addition, microbiological analyzes have shown that the magnetic field reduces total number of total germs of about 25\%.
\end{abstract}

Keywords: Static Magnetic field, microorganisms, raw milk

\section{Introduction}

The quality of drinking or irrigation water constitutes an enormous problem. In fact, improving the physicochemical characteristics of such substances is challenging, both for researchers, industrialists and farmers. Several methods of water treatment have been used.

However, they may present several disadvantages; for example, chemical techniques are costly, by the purchase of chemicals, while techniques based on membrane separation present a blockage problem and require maintenance and cleaning of the membranes. In addition, their effectiveness is selective because it does not capture the boron. In this context, magnetic treatment is seen as a process for treating water in industries, households, agriculture, etc. The magnetic devices are environmentally friendly, with low installation cost and they do not need energy (Hozaynet al., 2010). The magnetic treatment of water is based on the principle of "magnetic", where the electric energy is added, by a magnetic field, to the charged particles in water containing ions and small molecules with solid electrostatic charges. Energy is produced by the pulse of the particle permanently attached to the molecules of the surface energy (Gehret al., 1995).

The efficiency of the magnetic treatment changes according to several parameters such as; water flow, the quality of the treated water and even the initial conditions of the sample.

The magnetic field applied to water has been found as a treatment. In fact, the results obtained for natural

*Corresponding author:Anis Elaoud

DOI: http://Dx.Doi.Org/10.14741/Ijae/5.4.1 waters show that it can reduce the thickness of the scale deposited on hot surfaces and protect the pipes and structures containing water from a complete scale, while extending their lifetimes (Arabshahi, 2010). Bali et al., (2016) investigated the influence magnetic treatment on the precipitation of calcium carbonate.

Their study showed that the scaling potentiality of the treated water was significantly inhibited by the applied magnetic field. The anti-scaling properties using controlled precipitation tests confirmed that the magnetic field produces a "memory effect" roughly three days following the magnetic treatment. In another recent study, it has been reported that magnetic treatment of irrigation water can be used as an effective mean for softening the soil water. In fact, when applied to water, it leads to reduction of salt accumulation at the $30-60 \mathrm{~cm}$ soil depth (Zlotopolski, 2017).

Maheshwari and Grewal (2009) conducted studies on celery and peas using magnetic treatments of recycled, saline and potable water. They improved the productivity of yields and increased the water productivity. Numerous studies conducted on plants as tomato, sun flower and onion, irrigated with magnetized water, revealed the enrichment of plants seeds with Calcium, Iron, Potassium and Zinc which led to increased production quality (Elshokali and Abdelbagi, 2014). It has been found that the application of a magnetic field Enhances growth, chemical constituent and yield of chickpea (Hozayn et al, 2010). Maffei (2014) gave an apercu on the literature describing the positive effects of weak magnetic fields on plants such as Sunflower (Helianthus annuus), Pea (Pisumsativum), broad bean 
(Viciafaba) seedlings, soybean (Glycine max),... In his review, Maffei (2014) exposed several studies on plants responses by considering the effects of magnetic field even higher or lower than the geomagnetic fields. The main studied responses were development, transition to flowering, germination, redox status, photosynthesis.

Katsenioset al., (2016) used pulsed magnetic field for three time durations as a pre-sowing treatment in two cultivars of durum wheat seeds for three years experimentation. The results obtained showed a positive impact on agronomic characteristics enhancing germination, tillering, photosynthetic rate, dry weight, leaf area, stomatal conductance, chlorophyllcontent, transpiration rate and the wheat yield. In addition, enzyme measurements such $\alpha$ amylase activity was affected which could explain the improved germination.

Such experimentation was confirmed by other studies on other plants families, In fact, the effect of static magnetic water treatment on soybean and maize seedlings in salt stress condition enhanced early growth characteristics of all parameters associated with germination and also $\alpha$ - amylase and protease activities (Katariaet al., 2017). Recently, other studies on Amaranthaceae family revealed the positive impact of the magnetically treated water on the seed germination and precocious incubation period and the increases weight of the seeds. Which is a clear indication for the rapid penetration of water molecules into seeds (Krishnarajet al., 2017).

Many studies have already been investigated on microorganisms showing that magnetic field has no adverse effects on the cell. In fact no genomic or phenotypic alteration were observed when weak electromagnetic fields are applied on Escherichia coli and in other bacteria studies, it has been shown that with 8000 Gauss magnetic field strength, do not affect the enzyme catalase directly but it catalyses breakdown of hydrogen peroxide which contributes to dissolved oxygen (Ueno and Iwasaka,1996). Roshanzamir and Kakhki (2017) studied the effect of magnetic treatment on uropathogenic Escherichia coli growth provided from infected patients and a reference strain. The survival rate measured in the presence and absence of the magnetic field over time showed no statistically difference between both groups which presented a significant decline in survival rate when exposed to 18 and 20 Gauss.

The microbes such as Streptococcus parasanguinis, Staphilococcus, epidermidis, Rhodococcus, equi and Candidaalbicans were isolated from dental plaque deposit and the intensity of microbial growth on the basis of the modified optical density of agar turbidimetry assay, microbial count and spectrophotometry were applied.

The study was carried out using micromagnets and positive effect of the magnetic field, resulting in the significant reduction of all isolated microbes (Brkovicet al., 2015). Krepelkaet al., 2013 studied the effects of
Homogeneous and gradient stationary magnetic fields on the growth, morphology, and chemical composition of colonies of bacteria (Lactobacillus acidophillus, Staphylococcus epidermidis, Enterococcus durans, and Escherichia coli). Compared with a reference sample, the treated bacterium exhibited changes in the compound concentration and the ratio of proteins decreased.

Although the effect of magnetic fields is controversial, many studies confirmed the effect of magnetic field on microorganisms and plants and numerous papers which focused on the biological effects of the intensity of the magnetic field on bacteria and plant were published.

So far effect of its effect on drinking or irrigation water was very well studied; however its utilization remains promising in food industry as one of 'minimal' processing techniques that have limited impacts on nutritional and sensory properties of foods (Meyer et al., 2002). The objective of this study was to investigate the effect of magnetic field on some characteristic of row milk and to follow its bacterial evolution over the time until its spoilage. The output of this paper is as follows, in the second section we give the material and method used in this study in the third section we elucidate the results and finally we discuss the results and conclude.

\section{Materials and methods}

The present study was carried out to evaluate:

\section{*The effect of static magnetic field on some Chemical properties of raw milk}

The $\mathrm{pH}$, Dornic acidity and electric conductivity (EC) were measured at different time intervals ranging from 0 to 360 minutes. The milk parameters have been measured every thirty minutes and the reading of each parameter is replicated three times. The EC in $(\mathrm{dS} / \mathrm{m})$ was measured by an EC meter (model HI9812).

The $\mathrm{pH}$ values of the magnetized milk samples were measured by a digital $\mathrm{pH}$ meter (model BP3001).Titrable Acidity measurement was performed by Acid-base titration of the samples with a hydroxide solution of sodium $\mathrm{NaOH}(\mathrm{N} / 9) 0.1 \mathrm{~N}$ in the presence of phenophthalein as colored indicator. The titration was carried out by means of a small titrous burette of magnetic agitation until pinkish coloration.

\section{*The effect of static magnetic field on total germs of raw milk}

We follow the total germs in untreated (naturally fresh) raw milk and magnetically treated milk. For that, we divided our milk sample into two batches. A lot remains in the raw state and the other lot will undergo a magnetic treatment with 3300 Gauss for 60 minutes. The batches are Kept cold $\left(4^{\circ} \mathrm{C}\right)$. The development of total germs is examined, in the two 
types of milks, every two days interval for 6 days. All experiments were run in triplicates and the temperature of the milk before each seeding was moderated to ambient temperature $\left(24.8^{\circ} \mathrm{C}\right)$.

\subsection{Statistical Analysis}

The results data were submitted to an analysis of variance at a $5 \%$ significance level. The effects of the variables were studied by regression analysis. All statistical analyses were performed using the Excel specific program (Statistical). The analysis based on the coefficient of determination, the significance of regression coefficients. The significance of coefficients was estimated by student's t-test and p-values. The higher the level of the t-value and the lower the pvalue, the more significant is the coefficient (Khuriet al., 1987).

The sign of each coefficient suggests the direction of the relationship. The elimination of insignificant variables gives more accurate forecasts according to Sonmez and Rowings (Sonmezet al., 1998).

The $\mathrm{R}^{2}$ have been widely used for model evaluation, these statistics are oversensitive to high extreme values and insensitive to additive and proportional differences between model predictions and measured data (Legates et al., 1999).

\section{Results and discussion}

\section{*Chemical Parameters}

It is observed that the $\mathrm{pH}$ value remains constant for 6 hours after the magnetic treatment (Figure 1). The same facts are observed for Dornic acidity of milk that remains almost constant after the magnetic treatment (about $105^{\circ} \mathrm{D}$ ).

As it is known, titratable acidity is a better predictor of acid's impact on flavor than pH (Sadler and Murphy, 2010). This study didn't demonstrate any change in the $\mathrm{pH}$ nor in the titratable acidity which indicates that the applied magnetic field didn't alter the flavor of the milk.

However, the curve for monitoring the electrical conductivity of milk is characterized by a drop in the conductivity value just after a few minutes of treatment and then followed by a gradual increase to the initial value (fig 2).

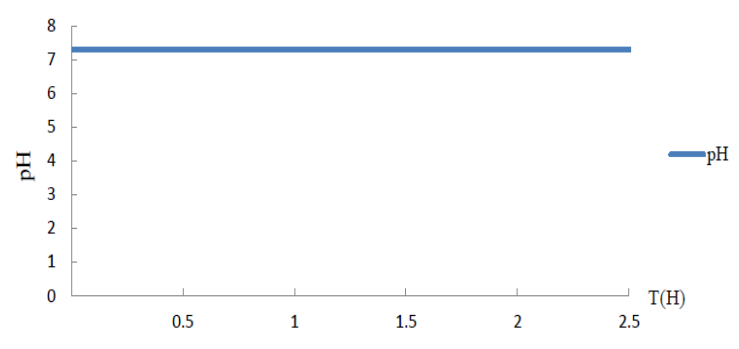

Fig. 1 pH curve of a magnetized milk sample

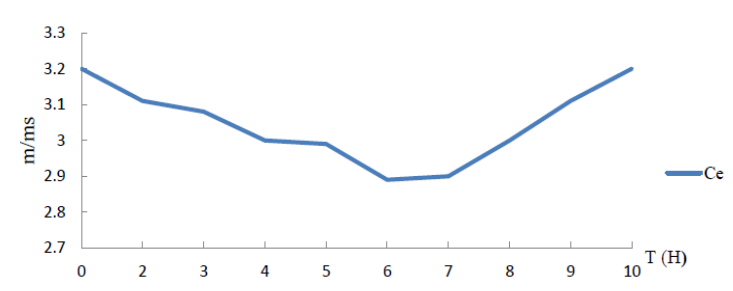

Fig. 2 Electrical conductivity curve of a magnetized milk sample

\section{${ }^{*}$ Microbiological analysis}

After incubation of the dishes, the colonies that grew on the agar were counted. It was found that the number of total germs in $1 \mathrm{ml}$ of fresh untreated milk increases day after day until the total alteration of the sample. This increase is observed too for fresh milk treated by a magnetic field but it is reduced (don't exceed $1700 \mathrm{UFC} / \mathrm{ml}$ versus $3000 \mathrm{UFC} / \mathrm{ml}$ for row milk) (Figure 3). For the dilution 2 and 3, it was observed through the time, a decrease in the treated samples (Figures 4 and 5). For the $8^{\text {th }}$ day the dishes of row milk were with uncountable bacteria, however with treated milk, the bacteria number remains countable $(\mathrm{N}=23.41$ $10^{2} \mathrm{UFC} / \mathrm{ml}, \mathrm{N}=21.0110^{3} .2 \mathrm{UFC} / \mathrm{ml}$ and $\mathrm{N}=22.1110^{4}$ $\mathrm{UFC} / \mathrm{ml}$ for dilution 1,2 and 3 , respectively). These results showed that the total number of total germs is reduced in milk treated with magnetic fields. However it increases in non treated milk samples. Besides, total germs in fresh milk begin to resist and develop before those which are found in the magnetized milk, thus a degradation of magnetic effect which produces the end of the phenomenon. To decide whether the magnetic field reduces the total bacterial load over time compared to untreated raw milk a student test analysis was used. Results are given by the Table1 which indicates that the magnetized field has decreased total germs. This result is very significant p-values were $<0.05(7,662 \mathrm{E}-05,0,006,0,048$ for dilution 1,2 and 3 , respectively).

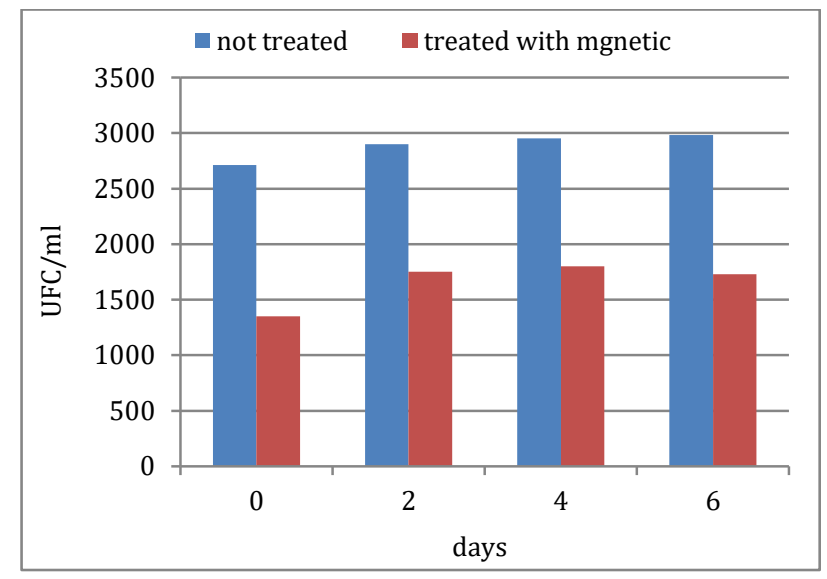

Fig. 3 Diagram of the number of total germs for the first dilution 


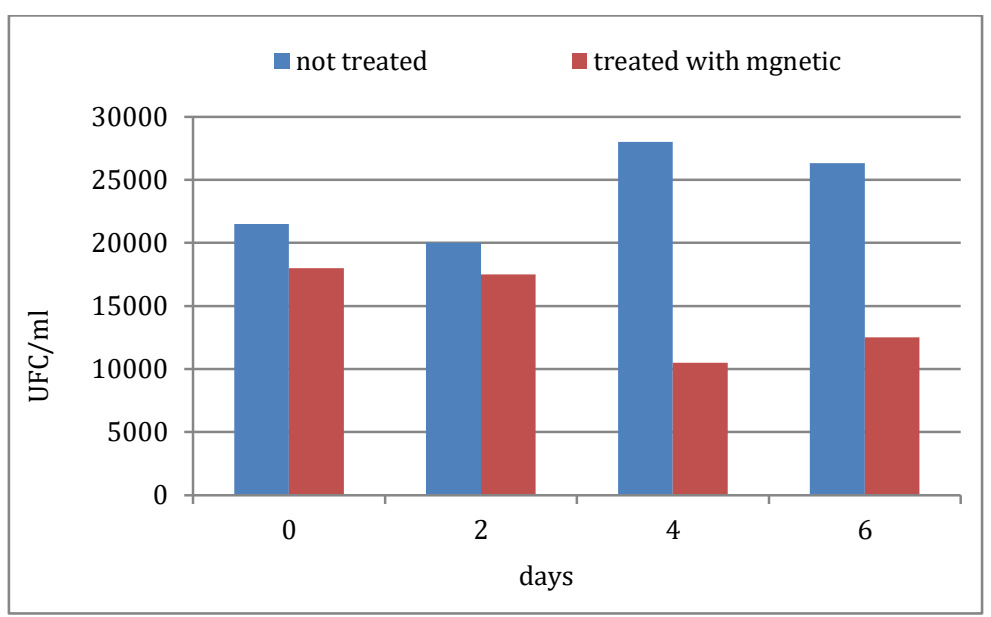

Fig. 4 Diagram of the number of total germs for the second dilution

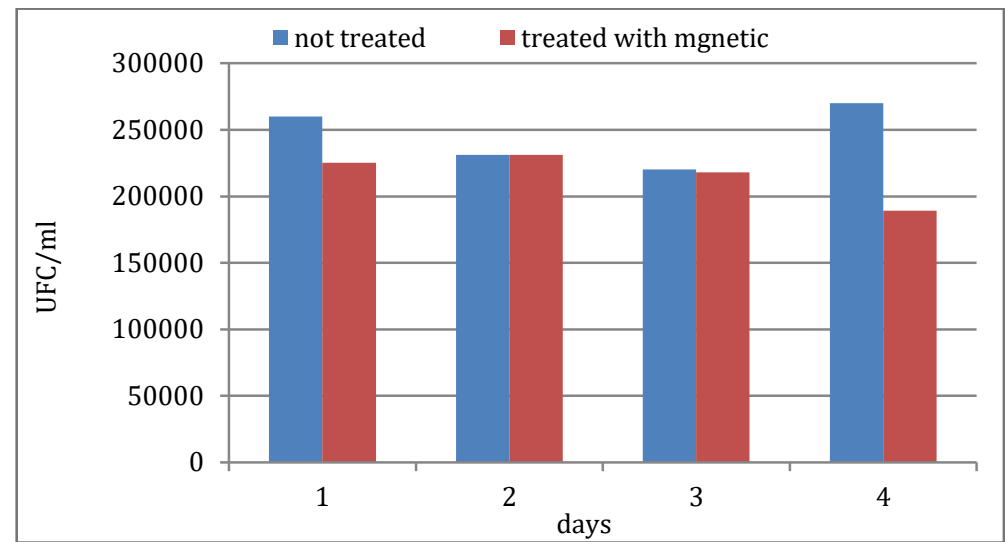

Fig.5 Diagram of the number of total germs for the third dilution

Table1. Student tests table for means of total germs in raw (not treated) and magnetic treated milks

\begin{tabular}{|c|c|c|c|}
\hline & & Not Treated & Treated with mgnetic \\
\hline & Mean & 2886,0225 & 1658,5225 \\
\hline \multirow[t]{6}{*}{ Dilution 1} & Variance & 14699,366 & 42889,48343 \\
\hline & Student $t$ & 10,230 & \\
\hline & Critical $t$ value & 2,015 & \\
\hline & $P$ value & $7,662 \mathrm{E}-05$ & \\
\hline & & Not Treated & Treated with mgnetic \\
\hline & Mean & 23960,2 & 14635,2 \\
\hline \multirow[t]{5}{*}{ Dilution 2} & Variance & 14509466,67 & 13729633,34 \\
\hline & Student $t$ & 3,509 & \\
\hline & Critical $t$ value & 1,943 & \\
\hline & $P$ value & 0,006 & \\
\hline & & Not Treated & Treated with mgnetic \\
\hline \multirow[t]{5}{*}{ Dilution 3} & Mean & 245326,5 & 215851,75 \\
\hline & Variance & 555882935 & 346263167,6 \\
\hline & Student $t$ & 1,962 & \\
\hline & Criticalt value & 2,446 & \\
\hline & $P$ value & 0,048 & \\
\hline
\end{tabular}




\section{Conclusion}

In this work, we applied a static magnetic treatment of 12 Tesla' strength for 1 hour to fresh non-sterilized milk and we followed some chemical and microbial parameters through the time. The magnetic treatment preserved the $\mathrm{pH}$ and the acidity of the milk, however it reduced its conductivity and in addition, microbiological analyzes have shown that magnetic reduces total number of total germs of about $25 \%$. Such study could be a perspective to the field of food preservation where emergent technologies try to respond to the growing consumers demand for techniques of bacterial inactivation without having to use strong heat destruction or treatment. This demand encourages more investigations of magnetic fields as minimal processing technology in the food industry.

\section{References}

H. Arabshahi(2010). The Magnetic Protection of Cooling System in Internal Combustion Engines against Corrosion and Scale Formation. Universal An International Journal of Chemistry,Vol 1(1), 336

M. Bali, Y. Mabrouk and N. Abdelli (2016). Effects of magnetic treatment on scaling power of hard waters.Separation and Purification Technology, 171, 88-92.

L. BasantMaheshwari, S.G. Harsharn, (2009). Magnetic treatment of irrigation water: Its effects on vegetable crop yield and water productivity, In Agricultural Water Management, Volume 96, Issue 8, Pages 1229-1236.

S. Brkovic,S. Postic and D. Ilic (2015). Influence of the magnetic field on microorganisms in the oral cavity. J Appl Oral Sci. 23(2): 179-186.

A.M. Elshokaliand A.M. Abdelbagi (2014). Impact of magnetized water on elements contents in plants seeds. International Journal of Scientific Research and Innovative Technology, Vol. 1 No. 4.

R. Gehr, Z. A. Zhai, J. A. Finch, and S. R. Rao (1995). Reduction of soluble mineral concentrations in $\mathrm{CaSO} 4$ saturated water using a magnetic field. Water Research, 29, Issue 3. p. 933-940.

M. Hozayn and A. Mohamed and S. Abdul Qados (2010). Irrigation with magnetized water enhances growth, chemical constituent and yield of chickpea (Cicerarietinum L.). Agriculture and biology journal of northAmerica, 2151 7525.

N. Katsenios, D. Bilalis, A. Efthimiadou, G. Aivalakis, A.E Nikolopoulou, A. Karkanis and I.Travlos (2016). Role of pulsed electromagnetic field on enzyme activity, germination, plant growth and yield of durum wheat, In Biocatalysis and Agricultural Biotechnology, Vol. 6, 152158.

P. Krepelka, E. Hutova, and K. Bartusek (2013). Effect of Stationary Magnetic Fields on Different Bacterial Strains.PIERS Proceedings. Stockholm, Sweden.12-15.

C. Krishnaraj, S.I Yun and V.K. Abhay Kumar (2017). Effect of Magnetized Water (Biotron) on Seed Germination of Amaranthaceae Family ChandranJournal of Academia and Industrial Research (JAIR) Volume 5, Issue 10. ISSN: 22785213

E. Massimo Maffei (2014). Magnetic field effects on plant growth, development, and evolution.FrontPlant Sci. 2014 5: 445 .

A.S. Meyer, K.I. Suhr, P. Nielsen and F. Holm (2002).Natural food preservatives. In: Ohlsson.T. andBengtsson. Minimal processing technologies in the food industry.Cambridge, England Woodhead.pp. 124-174.

S. Mousavian-Roshanzamir and A. Makhdoumi-Kakhki (2017). The Inhibitory Effects of Static Magnetic Field on Escherichia coli from two Different Sources at Short Exposure Time. Rep BiochemMol Biol. 2017 Apr; 5(2): 112116.

G.D. Sadler,P.A. Murphy (2010). pH and Titratable Acidity. In: Food Analysis. Food Analysis. Springer, Boston, MA

K. Sunita, LokeshBaghel, K.N. Guruprasad (2017). Pretreatment of seeds with static magnetic field improves germination and early growth characteristics under salt stress in maize and soybean, In Biocatalysis and Agricultural Biotechnology, Vol.10, Pages 83-90.

S. Ueno and M. Iwasaka (1996). Catalytic activity of catalase under strong magnetic fields of up to 8T. J. Appl. Phys., 79 . 4705-4707. 